\title{
Acessibilidade aos serviços básicos de saúde: um caminho ainda a percorrer
}

\author{
Access to Primary Healthcare Services: still a way to go
}

Antônio da Cruz Gouveia Mendes ${ }^{1}$

Gabriella Morais Duarte Miranda ${ }^{1}$

Karla Erika Gouveia Figueiredo ${ }^{1}$

Petra Oliveira Duarte ${ }^{1}$

Betise Mery Alencar Sousa Macau Furtado ${ }^{1}$

${ }^{1}$ Departamento de Saúde

Coletiva, Centro de

Pesquisas Aggeu

Magalhães, Fundação

Oswaldo Cruz. Campus da

UFPE, Av. Moraes Rego s/n

Atrás da Biblioteca da

Saúde, Cidade

Universitária. 50670-420

Recife PE.

antoniodacruz@gmail.com

\begin{abstract}
This study seeks to evaluate accessibility to the Basic Units of the Family Health Strategy (ESF-UB) and Traditional Basic Units (BU-T) in the city of Recife in 2009. Data were collected through three instruments: a roadmap for systematic observation of the units and questionnaires for users and professional units. This is a descriptive cross-sectional study using a quantitative approach, and 1180 users, 61 doctors and 56 nurses were interviewed. The results showed good ties and recognition of users whereby primary healthcare is seen as the access portal to the health system. In the comparison between ESF-UB and UB$T$, evaluations are always more favorable to the family healthcare strategy, though with relatively insignificant differences. The overall result revealed widespread dissatisfaction with the difficulty of obtaining drugs and taking tests, and also with the waiting times and access to specialized care. This showed the existence of organizational problems that may constitute barriers limiting accessibility to basic healthcare services for users.
\end{abstract}

Key words Primary healthcare, Accessibility, Health evaluation
Resumo Este estudo tem por objetivo avaliar a acessibilidade nas Unidades Básicas da Estratégia de Saúde da Família (UB-ESF) e Unidades Básicas Tradicionais (UB-T) da cidade do Recife em 2009. Os dados foram coletados através de três instrumentos: roteiro de observação sistemática das unidades e questionários destinados aos usuários e profissionais das unidades. Trata-se de um estudo descritivo de corte transversal com abordagem quantitativa. Foram entrevistados 1.180 usuários, 61 médicos e 56 enfermeiros. Os resultados indicaram uma boa vinculação e adscrição dos usuários evidenciando a atenção básica como porta de entrada do sistema de saúde. Na confrontação entre UB-ESF e UB-T as avaliações são sempre favoráveis à estratégia de saúde da família, diferenças nem sempre significativas. O resultado geral evidenciou uma grande insatisfação geral com a facilidade para obter medicamentos, realizar os exames, com o tempo de espera e o acesso à referência especializada, demonstrando a existência de problemas organizacionais que podem se constituir em barreiras que limitam a acessibilidade dos usuários aos serviços básicos de saúde.

Palavras-chave Atenção básica, Acessibilidade, Avaliação em saúde 


\section{Introdução}

A implantação do SUS trouxe, em 1988, a mudança do modelo de atenção à saúde até então existente. De um modelo hospitalocêntrico e de caráter curativo, passou-se a um modelo voltado para as ações de prevenção e de promoção da saúde, baseado em atividades principalmente coletivas $^{1}$. Desde então, a universalização do acesso, a integralidade e a territorialização orientam o sistema de saúde.

O modelo de atenção à saúde do Brasil é orientado pelas diretrizes da atenção básica, e tem a Saúde da Família como estratégia prioritária para sua organização e expansão, proporcionando maior acesso e resolubilidade a partir do trabalho de equipes que assumem a responsabilidade pela população de uma área geográfica definida onde são desenvolvidas ações individuais e coletivas de forma integral e contínua ${ }^{2}$.

Acesso é considerado um dos atributos essenciais para o alcance da qualidade nos serviços de Saúde ${ }^{3}$, no entanto é um conceito complexo que varia entre diversos estudiosos, inclusive na sua terminologia. Alguns utilizam o termo acesso, outros o termo acessibilidade, sendo eles muitas vezes empregados de forma ambígua e intercalados ${ }^{4}$.

Para Donabedian ${ }^{5}$ acessibilidade é algo adicional à mera presença ou disponibilidade do recurso em um dado momento e lugar, podendo ser distinta em duas classes: a sócio-organizacional e a geográfica. A primeira refere-se aos aspectos estruturais (funcionamento) dos serviços que interferem na relação destes com os usuários, por exemplo, o tempo de espera para o atendimento. São recursos de caráter não espacial que facilitam ou obstaculizam os esforços do cliente para obter atenção. A segunda pode ser medida pela distância e tempo de locomoção, custo da viagem, dentre outros. Travassos ${ }^{4}$ afirma que a mencionada definição avança na abrangência do conceito já que a ele acrescenta-se a adequação dos profissionais e dos recursos às necessidades da população.

Starfield ${ }^{6}$ destaca que a acessibilidade constitui-se no elemento estrutural necessário para que se atinja a atenção ao primeiro contato, à medida que possibilita a chegada das pessoas aos serviços. Representa um importante componente de um sistema de saúde no momento em que se efetiva o processo de busca e obtenção do cuidado ${ }^{7}$.

Fatores ligados à acessibilidade sócio-organizacional (tempo de espera para a marcação de consulta e para o atendimento, facilidade na rea- lização de exames e aquisição de medicamentos) e geográfica (distância da casa ao serviço) são considerados atributos essenciais para a garantia da qualidade na atenção básica ${ }^{8}$. Além desses atributos, a Educação em Saúde é também um aspecto envolvido com a qualidade na atenção básica $^{9}$

Todavia o acesso aos serviços de saúde no Brasil tem sido relatado na literatura como um dos principais problemas relacionados à assistên$\mathrm{cia}^{10}$. Essas dificuldades podem estar associadas tanto às características do atendimento quanto às barreiras organizacionais e geográficas ${ }^{7}$, o que contribui, na maioria das vezes, para a procura pelos cidadãos por planos privados de saúde ${ }^{11}$.

O conceito social, como princípio do SUS, implica a possibilidade dos usuários intervirem nos serviços de saúde, tanto na proposição de políticas e ações quanto na finalização de sua efetivação. Neste sentido, seu fortalecimento no âmbito do SUS e o incentivo à participação comunitária, em particular na atenção básica à saúde, pressupõem a concepção do usuário como corresponsável pela gestão do sistema de saúde e com competência para avaliá-lo, bem como para nele intervir e modificá- $\mathrm{lo}^{12}$.

Destaca-se aqui o usuário como principal qualificador do Sistema de Saúde ao expressarem suas preferências, expectativas, satisfação ou insatisfação, acerca dos métodos, circunstâncias e resultados da atenção ${ }^{13-16}$.

A avaliação da acessibilidade, na perspectiva dos usuários deve levar em consideração os atores envolvidos, principalmente os profissionais finalísticos do sistema de saúde, portanto, a percepção dos profissionais de saúde é de fundamental importância para o aprimoramento das propostas voltadas ao setor. Além dos usuários, Vilela ${ }^{17}$ enfatiza a importância da percepção dos profissionais de saúde para o aprimoramento das propostas voltadas ao setor.

Este estudo teve como objetivo realizar uma avaliação comparativa sobre a acessibilidade entre as Unidades Básicas da Estratégia Saúde da Família (UB-ESF) e as Unidades Básicas Tradicionais (UB-T) da cidade do Recife no ano de 2009.

\section{Métodos}

Trata-se de um estudo descritivo de corte transversal, a partir de dados primários, desenvolvido dentro de uma abordagem quantitativa.

Para avaliar a acessibilidade, foram realizadas entrevistas com usuários e profissionais das 
unidades de saúde da família e unidades básicas de saúde, além de observação sistemática.

Em 2009, a rede ambulatorial básica de saúde na cidade do Recife era composta por 24 Unidades Básicas Tradicionais (UB-T) e 112 Unidades Básicas da Estratégia de Saúde da Família (UBESF) com 240 Equipes de Saúde da Família, representando uma cobertura de $54 \%$ da população.

Para definição da amostra dos profissionais foi realizada uma amostragem estratificada proporcional considerando uma fração representativa de $20 \%$ do universo de profissionais para as Unidades da Saúde da Família. Dessa forma, definiu-se pela participação de 48 equipes de saúde da família (ESF), ou seja, 96 profissionais médicos e enfermeiros. Nas 24 unidades básicas de saúde tradicionais existiam 100 médicos e 15 enfermeiros lotados, sendo entrevistados 23 e 8 respectivamente, escolhidos de forma aleatória.

Para avaliação da acessibilidade com os usuários as entrevistas foram realizadas após atendimento nas unidades selecionadas. Para cálculo da amostra, foi mensurado o potencial de atendimento semanal dos médicos e enfermeiros das UB-ESF e apenas médicos das UB-T, pois nestas últimas unidades o enfermeiro não tem o mesmo perfil de atendimento finalístico. Sendo assim, definiu-se por amostrar 960 usuários nas unidades de saúde da família e 220 nas unidades tradicionais, o que equivale a capacidade de atendimento semanal de aproximadamente $3 \%$ e $3,5 \%$, respectivamente. Tal amostra é suficiente para determinar diferenças de proporções na ordem de 0,1 com grau de confirmação de $95 \%$ e poder estatístico maior que $80 \%$.

A coleta de dados foi realizada por oito bolsistas, estudantes do último ano do curso de enfermagem da Universidade de Pernambuco (UPE). Optou-se pela seleção destes, pela sua familiaridade com a Política Nacional de Atenção Básica e a Estratégia de Saúde da Família facilitando assim a compreensão das questões postas aos entrevistados, bem como a familiaridade com a metodologia científica. Os estudantes passaram por um treinamento para conhecer os objetivos da pesquisa e o instrumento a ser aplicado.

O questionário aplicado aos usuários, continha questões fechadas a serem avaliadas a partir da atribuição de um valor de 0 a 10 , formato baseado na escala Likert, de forma que para a avaliação de cada uma das questões contidas no questionário foi calculada a média por indivíduo e para a das categorias foi mensurada a média das questões contidas na categoria.

Nos estudos de satisfação, apesar da diversidade de métodos e instrumentos, a satisfação é em geral estabelecida como boa ou muito boa; alta ou muito alta ${ }^{18,19}$ ou quando a avaliação obtém uma nota igual ou superior a sete ${ }^{20}$. Desta forma, considerando que a média geral das notas obtidas neste estudo foi 7,03, definiu-se como satisfação, as avaliações com notas iguais ou superiores a sete.

Para avaliação da percepção dos médicos e enfermeiros quanto às condições ofertadas para garantia da acessibilidade, foi solicitado ao profissional que conceituasse cada item como ótimo, bom, regular, ruim ou péssimo. Em seguida, para facilitar a análise, as categorias foram agrupadas em bom (ótimo e bom), regular e ruim (ruim e péssimo).

$\mathrm{Na}$ observação sistemática foram mensurados os tempos de espera na unidade para ser atendido e o tempo de realização da consulta, sendo registrados em formulário próprio.

Para verificar a existência de diferenças significativas na distribuição das frequências entre as unidades, utilizou-se o teste de Qui-quadrado, com correção de Yates ou Teste Exato de Fisher quando da existência de caselas com valor esperado inferior a cinco. Para todos os testes, o nível de significância adotado foi de 5\%.

Esta pesquisa foi aprovada pelo Comitê de Ética e Pesquisas em Seres Humanos do Centro de Pesquisas Aggeu Magalhães. Para a aplicação dos questionários foi apresentado aos entrevistados o Termo de Consentimento Livre e Esclarecido, contendo os objetivos da pesquisa e esclarecimentos quanto à participação voluntária, à garantia do direito de não identificação como também à possibilidade de a qualquer momento não mais fazer parte da pesquisa, e da existência de danos ou riscos mínimos à população envolvida.

\section{Resultados}

Na avaliação do vínculo do usuário com a UBESF e com a UB-T (Tabela 1), 86,2\% e 92,2\%, respectivamente, citaram que na ocorrência de um problema de saúde procuram os referidos serviços para realização de atendimento, assim como, aproximadamente $95 \%$ já tinham sido atendidos nas unidades em outros momentos.

No tocante à estratégia utilizada para a marcação da consulta, observa-se um predomínio da marcação na própria unidade de saúde, sendo superior entre os frequentadores das UB-T (88,8\%) em relação aos da UB-ESF (72,5\%). Entretanto, 22,9\% dos agendamentos nas UB-ESF são efetuados pelos Agentes Comunitários de 
Saúde (ACS), enquanto que nas UB-T esse valor reduziu-se a metade $(11,8 \%)$.

A maioria dos frequentadores das UB-ESF $(43,7 \%)$ e das UB-T $(79,6 \%)$ consegue agendamento em até uma semana, e ainda 19,2\% e 21,3\% deles, respectivamente, são atendidos no mesmo dia. Salienta-se que boa parte dos usuários das UB-ESF (26,0\%) espera mais de 15 dias para conseguir ser atendido após marcação da consulta. Esse percentual mostrou-se inferior $(13,9 \%) \mathrm{em}$ relação ao tempo esperado nas UB-T.

O nível de saúde autorreferido dos usuários foi bom ou regular tanto nas UB-T $(95,5 \%)$ quanto nas UB-ESF $(90,5 \%)$ e aproximadamente $60 \%$ dos usuários relataram não tomar nenhum medicamento de uso contínuo no momento.

Considerando as três dimensões que compõem as condições de atendimento sob a ótica do usuário (Tabela 2), apreende-se que a melhor avaliação feita refere-se à distância entre a casa $\mathrm{e}$ a unidade, sendo que nas UB-ESF as pessoas mostraram-se mais satisfeitas $(80,4 \%)$ em relação às UB-T $(71,6 \%)$, apresentando significân- cia estatística. A maior insatisfação esteve ligada as facilidades para o atendimento, sendo superior nas UB-T $(71,2 \%)$ em relação às UB-ESF $(65,2 \%)$. Evidenciou-se ainda grande descontentamento com os tempos de espera, declarado por mais de 50,0\% dos usuários das unidades.

Analisando cada dimensão isoladamente, percebe-se na relacionada às facilidades de atendimento, que a possibilidade de participar de atividades educativas no momento de espera para a consulta obteve o mais alto percentual de insatisfação, sendo superior nas UB-T $(87,3 \%)$ comparando-se com as UB-ESF $(73,9 \%)$.

Tanto nas UB-ESF quanto nas UB-T nota-se elevada satisfação quanto à marcação de consulta na unidade $(54,7 \%$ e $56,8 \%)$, ao recebimento da medicação $(63,0 \%$ e 67,9\%) e finalmente quanto à facilidade para realização de exames $(64,6 \%$ e 66,8\%).

Por fim, quanto à facilidade para realização de agendamento de consultas especializadas, houve uma divergência entre os entrevistados, evidenciada pela insatisfação de $47,5 \%$ dos usuários

Tabela 1. Caracterização do Atendimento e Situação de Saúde dos Usuários atendidos nas UB-ESF e UB-T. Recife, 2009.

\begin{tabular}{|c|c|c|c|c|}
\hline \multirow[b]{2}{*}{ Situação de Saúde e Caracterização do Atendimento } & \multicolumn{2}{|c|}{ UB-ESF } & \multicolumn{2}{|c|}{ UB-T } \\
\hline & $\mathbf{N}$ & $\%$ & $\mathbf{N}$ & $\%$ \\
\hline \multicolumn{5}{|l|}{ Procura a unidade sempre que apresenta problema de saúde } \\
\hline Sim & 806 & 86,2 & 201 & 92,2 \\
\hline Não & 129 & 13,8 & 17 & 7,8 \\
\hline \multicolumn{5}{|l|}{ Primeira consulta na unidade } \\
\hline Sim & 41 & 4,4 & 11 & 5,0 \\
\hline Não & 897 & 95,6 & 211 & 95,0 \\
\hline \multicolumn{5}{|l|}{ Marcação de Consulta } \\
\hline Na unidade & 678 & 72,5 & 195 & 88,2 \\
\hline Com o ACS & 214 & 22,9 & 26 & 11,8 \\
\hline Outra & 43 & 4,6 & 0 & 0,0 \\
\hline \multicolumn{5}{|l|}{ Tempo para a realização da consulta } \\
\hline \multicolumn{5}{|l|}{ Não agendada } \\
\hline Mesmo dia & 175 & 19,2 & 46 & 21,3 \\
\hline \multicolumn{5}{|l|}{ Agendada } \\
\hline Até 7 dias & 398 & 43,7 & 172 & 79,6 \\
\hline 8 a 14 dias & 100 & 11,0 & 13 & 6,0 \\
\hline 15 a 30 dias & 207 & 22,7 & 29 & 13,4 \\
\hline Mais de 30 dias & 30 & 3,3 & 1 & 0,5 \\
\hline \multicolumn{5}{|l|}{ Nível de Saúde Autorreferido } \\
\hline Bom & 447 & 47,6 & 113 & 50,9 \\
\hline Regular & 403 & 42,9 & 99 & 44,6 \\
\hline Ruim & 89 & 9,5 & 10 & 4,5 \\
\hline \multicolumn{5}{|l|}{ Uso de medicamentos de forma contínua } \\
\hline Sim & 371 & 40,1 & 89 & 40,3 \\
\hline Não & 555 & 59,9 & 132 & 59,7 \\
\hline
\end{tabular}


das UB-ESF, contra 57,2\% de insatisfação nas UB-T, com diferença estatisticamente significante entre as unidades.

Considerando as questões relativas à dimensão tempo de espera, a única questão positivamente avaliada foi a duração da consulta, com satisfação superior a 80,0\% nas unidades. O tempo esperado para o recebimento dos resultados dos exames foi considerado insatisfatório por $63,0 \%$ e 61,3\% dos usuários das UB-ESF e UB-T. Na sequência, o tempo de espera para ser atendido também foi reprovado por $54,7 \%$ e $58,2 \%$ dos usuários das UB-ESF e UB-T, nesta ordem.
Analisando as dimensões que constituem as condições avaliadas pelos profissionais (Tabela 3), destaca-se inicialmente o fato de cerca 35,0\% dos profissionais das UB-ESF terem considerado ruim o número de famílias cobertas pelas ESFs.

A facilidade do acesso à unidade foi a dimensão melhor avaliada pelos profissionais dos dois tipos de serviços. Os profissionais das duas unidades avaliaram como regular a disponibilidade de exames e de medicamentos, tendo esta última dimensão apresentado significância estatística. Por fim, a disponibilidade de referências especializadas foi considerada a pior dimensão, sendo tal des-

Tabela 2. Avaliação da satisfação dos usuários em relação às condições de atendimento oferecidas nas UB-ESF e UB-T. Recife, 2009.

\begin{tabular}{|c|c|c|c|c|c|c|c|c|c|c|c|}
\hline \multirow[b]{3}{*}{ Condições de Atendimento } & \multicolumn{5}{|c|}{ UB-ESF } & \multicolumn{5}{|c|}{ UB-T } & \multirow{3}{*}{$\begin{array}{l}\mathrm{X}^{2} \text { Yates } \\
\mathrm{P} \text {-valor }\end{array}$} \\
\hline & \multicolumn{2}{|c|}{ Satisfeito } & \multicolumn{2}{|c|}{ Insatisfeito } & \multirow[b]{2}{*}{ Total } & \multicolumn{2}{|c|}{ Satisfeito } & \multicolumn{2}{|c|}{ Insatisfeito } & \multirow[b]{2}{*}{ Total } & \\
\hline & $\mathbf{N}$ & $\%$ & $\mathbf{N}$ & $\%$ & & $\mathbf{N}$ & $\%$ & $\mathbf{N}$ & $\%$ & & \\
\hline Distancia da casa & 755 & 80,4 & 184 & 19,6 & 939 & 159 & 71,6 & 63 & 28,4 & 222 & $\begin{array}{l}X^{2}=7,75 \\
p=0,005\end{array}$ \\
\hline Facilidades & 327 & 34,8 & 612 & 65,2 & 939 & 64 & 28,8 & 158 & 71,2 & 222 & $X^{2}=2,63$ \\
\hline Para marcar consulta na unidade & 512 & 54,7 & 424 & 45,3 & 936 & 126 & 56,8 & 96 & 43,2 & 222 & $\mathrm{p}=0,10$ \\
\hline Para participar de atividades educativas & 240 & 26,1 & 680 & 73,9 & 920 & 28 & 12,7 & 193 & 87,3 & 221 & \\
\hline Para pegar medicamentos & 575 & 63,0 & 337 & 37,0 & 912 & 142 & 67,9 & 67 & 32,1 & 209 & \\
\hline Para conseguir agendamento especializado & 385 & 52,5 & 348 & 47,5 & 733 & 77 & 42,8 & 103 & 57,2 & 180 & \\
\hline Para realizar exames & 509 & 64,6 & 279 & 35,4 & 788 & 127 & 66,8 & 63 & 33,2 & 190 & \\
\hline Tempo de Espera & 434 & 46,2 & 505 & 53,8 & 939 & 94 & 42,3 & 128 & 57,7 & 222 & $X^{2}=0,94$ \\
\hline Para ser atendido & 423 & 45,3 & 511 & 54,7 & 934 & 92 & 41,8 & 128 & 58,2 & 220 & $\mathrm{p}=0,33$ \\
\hline Duração da consulta & 799 & 86,0 & 130 & 14,0 & 929 & 159 & 82,4 & 34 & 17,6 & 193 & \\
\hline Para receber resultado & 291 & 37,0 & 495 & 63,0 & 786 & 74 & 38,7 & 117 & 61,3 & 191 & \\
\hline
\end{tabular}

Tabela 3. Avaliação pelos profissionais médicos e enfermeiros sobre as condições de atendimento oferecidas nas UB-ESF e UB-T. Recife, 2009.

\begin{tabular}{|c|c|c|c|c|c|c|c|c|c|c|c|c|c|}
\hline \multirow{3}{*}{ Condições de atendimento } & \multicolumn{6}{|c|}{ UB-ESF $(\mathrm{N}=92)$} & \multicolumn{6}{|c|}{ UB-ESF $(\mathrm{N}=92)$} & \multirow{3}{*}{$\begin{array}{c}\mathrm{X}^{2} \\
\text { P-valor }\end{array}$} \\
\hline & \multicolumn{2}{|c|}{ Ruim } & \multicolumn{2}{|c|}{ Regular } & \multicolumn{2}{|c|}{ Bom } & \multicolumn{2}{|c|}{ Ruim } & \multicolumn{2}{|c|}{ Regular } & \multicolumn{2}{|c|}{ Bom } & \\
\hline & $\mathbf{N}$ & $\%$ & $\mathbf{N}$ & $\%$ & $\mathbf{N}$ & $\%$ & $\mathbf{N}$ & $\%$ & $\mathbf{N}$ & $\%$ & $\mathbf{N}$ & $\%$ & \\
\hline Número de famílias cobertas & 32 & 34,8 & 29 & 31,5 & 31 & 33,7 & - & - & - & - & - & - & - \\
\hline Disponibilidade de medicamentos & 11 & 12,0 & 47 & 51,1 & 34 & 37,0 & 10 & 32,3 & 10 & 32,3 & 11 & 35,5 & $X^{2}=7,39$ \\
\hline Variedade de medicamentos & 30 & 32,6 & 31 & 33,7 & 31 & 33,7 & 10 & 32,3 & 9 & 29,0 & 12 & 38,7 & $\mathrm{p}=0,02$ \\
\hline Quantidade de medicamentos & 38 & 41,3 & 31 & 33,7 & 23 & 25,0 & 13 & 41,9 & 11 & 35,5 & 7 & 22,6 & \\
\hline Disponibilidade de exames & 33 & 35,9 & 41 & 44,6 & 18 & 19,6 & 11 & 35,5 & 15 & 48,4 & 5 & 16,1 & $X^{2}=0,22$ \\
\hline Exames laboratoriais & 36 & 39,1 & 27 & 29,3 & 29 & 31,5 & 10 & 32,3 & 11 & 35,5 & 10 & 32,3 & $\mathrm{p}=0,89$ \\
\hline Exames de imagem & 36 & 39,1 & 41 & 44,6 & 15 & 16,3 & 14 & 45,2 & 10 & 32,3 & 7 & 22,6 & \\
\hline Tempo do resultado de exames laboratoriais & 68 & 73,9 & 21 & 22,8 & 3 & 3,3 & 24 & 77,4 & 6 & 19,4 & 1 & 3,2 & \\
\hline Disponibilidade de referências especializadas & 53 & 57,6 & 26 & 28,3 & 13 & 14,1 & 20 & 64,5 & 10 & 32,3 & 1 & 3,2 & $X^{2}=2,74$ \\
\hline Facilidade de acesso a unidade & 4 & 4,3 & 24 & 26,1 & 64 & 69,6 & 3 & 9,7 & 7 & 22,6 & 21 & 67,7 & $\begin{array}{c}\mathrm{p}=0,25 \\
\mathrm{X}^{2}=2,11 \\
\mathrm{p}=0,34\end{array}$ \\
\hline
\end{tabular}


contentamento superior nas unidades tradicionais $(64,5 \%)$ em relação à saúde da família $(57,6 \%)$.

Considerando cada dimensão analisada, percebe-se que na disponibilidade de medicamentos a maior insatisfação esteve relacionada à quantidade dos medicamentos em ambos os serviços.

No tocante a disponibilidade de exames, a pior avaliação referiu-se ao tempo de recebimento dos resultados, o qual foi negativamente avaliado por $73,9 \%$ dos profissionais das UB-ESF e $77,4 \%$ das UB-T.

Sobre o tempo de espera para o atendimento (Tabela 4), mensurado durante a observação sistemática, observa-se duração superior na UB-T (89 minutos) em comparação com a UB-ESF (70 minutos), sendo a diferença estatisticamente significante entre as unidades. Além disso, a maioria dos usuários esperou entre 61 e 121 minutos para ser atendida, sendo $41,2 \%$ entre as UB-ESF e $34,6 \%$ entre as UB-T. Tempo superior a duas horas foi vivenciado mais do que o dobro de usuários nas UB-T $(24,4 \%)$ comparando-se com as UB-ESF (10,5\%).

Por fim, analisando o tempo de duração da consulta (Tabela 5), observou-se que os usuários das unidades de saúde da família foram atendidos em média durante 18 minutos, reduzindo para 13 minutos a duração dos atendimentos nas unidades tradicionais.

Nos dois tipos de unidades, a maioria das pessoas $(53,6 \%)$ permaneceu em atendimento entre 11 a 20 minutos. Chama a atenção, o fato de aproximadamente 40,0\% dos usuários das UBT permanecerem em atendimento por até $10 \mathrm{mi}$ nutos enquanto que nas UB-ESF o citado percentual é reduzido pela metade (20,3\%). Além

Tabela 4. Tempo de Espera do usuário nas unidades de Saúde da Família e Unidades Tradicionais, em minutos. Recife, 2009.

\begin{tabular}{|c|c|c|c|c|}
\hline Tempos de Espera & \multicolumn{2}{|c|}{ UB-ESF } & \multicolumn{2}{|c|}{ UB-T } \\
\hline Média & \multicolumn{2}{|c|}{70} & \multicolumn{2}{|c|}{89} \\
\hline \multicolumn{5}{|l|}{ Tempo de Espera } \\
\hline Faixa & $\mathbf{N}$ & $\%$ & $\mathbf{N}$ & $\%$ \\
\hline $1-10^{\prime}$ & 5 & 1,6 & 0 & 0 \\
\hline $11-30^{\prime}$ & 39 & 12,7 & 13 & 16,7 \\
\hline $31-60^{\prime}$ & 104 & 34,0 & 19 & 24,4 \\
\hline $61-120^{\prime}$ & 126 & 41,2 & 27 & 34,6 \\
\hline $121^{\prime}+$ & 32 & 10,5 & 19 & 24,4 \\
\hline Total & 306 & 100,0 & 78 & 100,0 \\
\hline
\end{tabular}

Teste Exato de Fisher $\mathrm{p}^{\star}$ valor $=0,01453$ disso, quase $25,0 \%$ dos usuários passaram mais de 20 minutos em atendimento na UB-ESF, o que é reduzido na UB-T (12,8\%).

\section{Discussão}

Os resultados indicaram uma boa vinculação e adscrição da população, demonstrados através da alta procura pelo serviço quando da ocorrência de problemas de saúde, além do que, aproximadamente $95 \%$ dos entrevistados afirmaram que não se tratava da primeira consulta nas unidades. A adscrição é reforçada pelo alto nível de satisfação dos usuários, principalmente os das UB-ESF, em relação à distância entre a sua casa e a unidade de saúde, o que evidencia que o papel da Estratégia de Saúde da Família, enquanto porta de entrada do sistema, está sendo cumprido.

Esses resultados corroboram com os de $\mathrm{Me}$ deiros et al. ${ }^{21}$ em que foi relatada alta procura pelos serviços básicos por serem resolutivos em relação aos problemas de saúde identificados. $\mathrm{O}$ acesso geográfico também foi considerado adequado pelos usuários nos estudos de Lima et al. ${ }^{10}$ e de Azevedo e Costa ${ }^{22}$.

Em relação ao agendamento da consulta, o percentual de 23,0\% de agendamento realizado pelos ACS para os usuários das UB-ESF, por um lado, pode demonstrar um desvio das ações de promoção da qualidade de vida e prevenção de doenças na comunidade; e por outro lado reforça a importância desse profissional na Estratégia de Saúde da Família. Medeiros et $\mathrm{al}^{21}$ constataram em seu trabalho que essa marcação é feita predominantemente pelo ACS (58,5\%), o que, segundo Trad et al..$^{23}$
Tabela 5. Tempo de Atendimento do usuário nas unidades de Saúde da Família e Unidades Tradicionais, em minutos. Recife, 2009.

\begin{tabular}{lrcrcr}
\hline Tempos de Atendimento & \multicolumn{2}{c}{ UB-ESF } & \multicolumn{2}{c}{ UB-T } \\
\hline $\begin{array}{c}\text { Média } \\
\text { Tempo de Atendimento }\end{array}$ & \multicolumn{2}{c}{$\mathbf{1 8}$} & \multicolumn{2}{c}{$\mathbf{1 3}$} \\
$\quad$ Faixa & \multicolumn{1}{c}{ N } & \multicolumn{1}{c}{$\%$} & \multicolumn{1}{c}{ N } & $\%$ \\
$1-10^{\prime}$ & 62 & 20,3 & 31 & 39,7 \\
$11-20^{\prime}$ & 160 & 52,3 & 37 & 47,4 \\
$21-30^{\prime}$ & 67 & 21,9 & 10 & 12,8 \\
$31-40^{\prime}$ & 12 & 3,9 & 0 & 0,0 \\
$41^{\prime}+$ & 4 & 1,6 & 0 & 0,0 \\
Total & $\mathbf{3 0 6}$ & $\mathbf{1 0 0 , 0}$ & $\mathbf{7 8}$ & $\mathbf{1 0 0 , 0}$ \\
& & & &
\end{tabular}

Teste Exato de Fisher $\mathrm{p}^{\star}$ valor $=0,003$ 
acarreta a grande satisfação dos usuários com o trabalho desenvolvido por tais profissionais.

Observou-se a predominância nas UB-ESF de um período de tempo superior a quinze dias para a obtenção da consulta na unidade após a marcação da mesma. Isso pode ser decorrente da característica desse serviço em atender uma demanda programada, como a realização de consultas de pré-natal que conforme recomendado pelo Ministério da Saúde devem ser realizadas nas mulheres grávidas uma vez ao mês. Por outro lado, o fato da maioria das pessoas $(79,6 \%)$ ser atendida em até sete dias nas UB-T pode evidenciar sua peculiaridade de acolher a demanda espontânea, o que pode explicar a alta satisfação do usuário para conseguir agendamento nesse tipo de serviço (56,8\%).

Similarmente Almeida e Macinko ${ }^{24}$ também descreveram em seu estudo certa tranquilidade para o agendamento de consulta na unidade principalmente nas UB-ESF, onde foi relatada mais frequentemente a possibilidade desse agendamento ocorrer no prazo de $24 \mathrm{~h}$.

Outras investigações indicaram dificuldades no agendamento de consultas que podem ser evidenciadas muitas vezes pela formação de filas para conseguir fichas para o atendimento $\mathrm{o}^{7,9,11,22,25}$. Cunha e Silva ${ }^{7}$ sinalizaram que este é o principal obstáculo encontrado nos serviços por eles investigados, o que pode gerar segundo Azevedo e Costa $^{22}$ desistência dos usuários em utilizarem os serviços prestados nas Unidades Básicas de Saúde.

$O$ fato da consulta na UB-ESF ser mais duradoura do que na UB-T corrobora com a concepção acima descrita de que em tal espaço deve existir um maior vínculo com os usuários, ao contrário do atendimento por demanda espontânea característico das UB-T, embora tais achados não sejam evidenciados na satisfação dos usuários. $\mathrm{O}$ tempo de consulta predominante de 20 minutos nos dois serviços mostrou-se coerente com o padrão estabelecido pelo Ministério da Saúde em $2001^{26}$, o qual estipula a realização de 04 consultas/hora para os médicos e 03 consultas/hora para enfermeiros, ou seja, aproximadamente 15 e 20 minutos, respectivamente. Ressalta-se, porém, que tal padrão deve ser ajustado conforme perfil epidemiológico, sazonalidade de cada microrregião, região ou estado, quando se fizer necessário.

Cotta et al. ${ }^{18}$ apontaram resultados semelhantes (15 a 30 minutos de consulta). Por outro lado, tempos inferiores a 10 minutos foram encontrados em outros estudos ${ }^{27-30}$.

As dificuldades para o atendimento foram maiores nas UB-T, o que decorre principalmente da insatisfação dos usuários em relação a possi- bilidade de participar de atividades educativas durante a espera para consulta e ao agendamento de consultas especializadas.

A insatisfação com o desenvolvimento de atividades educativas demonstram dificuldades na implementação das estratégias básicas preconizadas para a ESF. A Política Nacional de Humanização preconiza que a comunicação entre profissionais e usuários não deve acontecer apenas dentro dos consultórios, mas de uma forma permanente e continuada nos demais lugares da Unidade, resultados também encontrados em outros estudos ${ }^{9,21,24,31}$.

Alguns autores destacam que a sala de espera seria um local propício ao desenvolvimento de educação em saúde, além do que diminuiria o descontentamento com o tempo de espera beneficiando tanto os usuários quanto os profissionais em virtude da interação e troca de conhecimentos entre os mesmos ${ }^{12,32}$.

$\mathrm{O}$ descontentamento em relação às referências especializadas demonstra a baixa resolutividade da Atenção Básica, considerada porta de entrada para o sistema de saúde, implicando em baixo acesso dos usuários aos demais níveis da atenção.

A insatisfação por parte dos usuários em relação ao agendamento de consultas especializada foi compartilhada em outros trabalhos ${ }^{24}$. Além disso, Cunha e Vieira-da-Silva ${ }^{7}$ e Souza et $\mathrm{al}^{33}$ destacam que segundo a opinião dos usuários esse processo é mais dificultoso nas UB-T, já que nas UB-ESF eles contam com a ajuda do profissional para a priorização e encaminhamento dos casos mais graves. Por outro lado, o estudo de Medeiros et al. ${ }^{21}$ realizado em uma unidade Básica (UB) do Rio Grande do Norte comprovou alta satisfação em relação ao encaminhamento para referência.

Nota-se um bom julgamento dos usuários sobre a facilidade para realizar exames e recebimento de medicamentos, assim como bom julgamento dos profissionais quanto à disponibilidade de medicamentos e exames. Entretanto, constata-se também concordância entre a opinião dos usuários e profissionais em relação à insatisfação acerca do tempo de espera para o recebimento dos exames laboratoriais, demonstrando ser este um ponto frágil relacionado a dificuldades na organização do serviço e ao mesmo tempo à desarticulação entre o laboratório e o sistema como um todo.

Semelhantemente, a literatura tem apontado certa melhora acerca da realização dos exames requisitados, principalmente quando os mesmos 
são coletados na própria unidade. Por outro lado ainda persiste a questão da demora para o retorno dos resultados que muitas vezes é relatado como um dos principais problemas do acesso na percepção dos usuários ${ }^{10,22,24,33}$.

Assim como neste estudo, outros autores também evidenciaram facilidade em relação ao fornecimento dos remédios prescritos, em contrapartida a quantidade recebida não foi capaz de contemplar toda a demanda ${ }^{9,10,22,24,25,33}$.

Segundo a Política Nacional de Medicamentos, é dever do município assegurar o suprimento adequado de medicamentos básicos indispensáveis para atender as necessidades da população. Por outro lado é preciso avaliar se a prescrição médica mantém coerência com a padronização de medicamentos, a qual deve ser planejada e constantemente atualizada a partir da observação do contexto epidemiológico local. A inexistência dessa integração compromete a eficácia das ações governamentais nesse setor ${ }^{34}$.

Em relação ao tempo de espera para o atendimento, predominou nos dois tipos de unidade o intervalo entre uma a duas horas, sendo mais fácil esse tempo ocorrer nas UB-ESF (34\%) do que nas UB-T (24,4\%). Essa questão foi motivo de insatisfação relatada pela maioria das pessoas que frequentavam tais unidades, o que merece atenção especial já que a espera é o momento da vinculação do indivíduo com a unidade. A insatisfação dos usuários com o tempo de espera para atendimen-

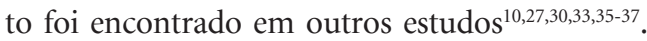

A recomendação de Starfield ${ }^{6}$ é que o período esperado não ultrapasse os 30 minutos. Almeida e Macinko ${ }^{24}$ também constataram que é mais fácil passar mais de trinta minutos esperando pela consulta na UB-ESF do que nas UB-T e que quase nunca as pessoas são atendidas em menos de 30 minutos nessas unidades.

Apesar da maior satisfação dos usuários das UB-ESF em todas as dimensões analisadas (distância da unidade, facilidades para o atendimento às suas necessidades no atendimento e o tempo de espera), as diferenças não foram estatisticamente significativas.

Os profissionais avaliaram melhor as condições ofertadas para garantia da acessibilidade quanto à disponibilidade de medicamentos, exames laboratoriais e de imagem, além da disponibilidade de referência às especialidades quando necessário nas UB-ESF. Assim como, foi constatada uma maior duração das consultas médicas e de enfermagem, o que sugere um maior cuidado no atendimento.

Essas pequenas diferenças de avaliação entre as UB-ESF e as UB-T a favor da primeira não devem esconder o alto grau de insatisfação geral com a facilidade para obter medicamentos, realizar os exames, com o tempo de espera e o acesso à referência especializada, demonstrando a existência de problemas organizacionais que podem se constituir em barreiras que limitam a acessibilidade dos usuários aos serviços básicos de saúde. Portanto, a estratégia de reorientação do modelo de saúde a partir da atenção básica precisa avançar, tendo ainda um caminho a percorrer para garantir uma maior acessibilidade da população e maior qualificação da atenção à saúde. 


\section{Colaboradores}

ACG Mendes trabalhou na concepção, coleta e sistematização de dados, delineamento e elaboração do texto. GMD Miranda trabalhou na concepção e sistematização de dados, delineamento e elaboração do texto. KEG Figueiredo trabalhou no delineamento e elaboração do texto. $\mathrm{PO} \mathrm{Du}$ arte trabalhou na concepção, coleta e sistematização de dados. BMASM Furtado trabalhou na concepção, coleta e sistematização de dados.

\section{Referências}

1. Valentim IVL, Kruel AJ. A importância da confiança interpessoal para a consolidação do Programa de Saúde da Família. Cien Saude Colet 2007; 12(3):777-788.

2. Brasil. Ministério da Saúde (MS). Secretaria de Atenção à Saúde. Departamento de Atenção Básica. Política nacional de atenção básica. Série A. Normas e Manuais Técnicos. Brasília: MS; 2006. (Série Pactos pela Saúde 2006, v. 4).

3. Mendes ACG. A delicadeza esquecida: Avaliação da qualidade das Emergências. Recife: Editora Universitária da UFPE; 2010.

4. Travassos C, Martins M. Uma revisão sobre os conceitos de acesso e utilização de serviços de saúde. Cad Saude Publica 2004; 20(Supl. 2):190-198.

5. Donabedian A. Los espacios de La Salud: Aspectos fundamentales de La organización de La atención médica. México DF: Fondo de Cultura Económica; 1988.

6. Starfield B. Atenção Primária: equilíbrio entre necessidades de saúde, serviços e tecnologia. Brasília: UNESCO, MS; 2002.

7. Cunha ABO, Vieira-da-Silva LM. Acessibilidade aos serviços de saúde em um município do Estado da Bahia, Brasil, em gestão plena do sistema. Cad Saude Publica 2010; 26(4):725-737.

8. Halfoun VLRC, Aguiar OB, Aguir OB, Mattos DS, Construção de um Instrumento para Avaliação de Satisfação da Atenção Básica nos Centros Municipais de Saúde do Rio de Janeiro. Rev. bras. educ. méd 2008; 32(4):424-430.

9. Serapioni M, Silva MGC. Avaliação da qualidade do Programa Saúde da Família em municípios do Ceará. Uma abordagem multidimensional. Cien Saude Colet 2011; 16(11):4315-4326.

10. Lima MADS, Ramos DD, Rosa RB, Nauderer TM, Davis R. Acesso e acolhimento em unidades de saúde na visão dos Usuários. Acta paul. Enferm 2007; 20(1):12-17.

11. Gerschman S, Veiga L, Guimarães C, Ugá MAD, Portela MC, Vasconcellos MM, Barbosa PR, Lima SML. Estudo de satisfação dos beneficiários de planos de saúde de hospitais filantrópicos. Cien Saude Colet 2007; 12(2):487-500.

12. Jorge MSB, Guimarães JMX, Vieira LB, Paiva FDS, Silva DR, Pinto AGA. Avaliação da qualidade do Programa Saúde da Família no Ceará: a satisfação dos usuários. Rev. baiana saúde pública 2007; 31(2): 256-266.

13. Organização Panamericana de Saúde (OPAS). Renovación de la Atención Primaria de Salud en las Américas. Brasil: OPAS; 2005.

14. Donabedian A. The international dimensiono of quality assement and assurance. Salud Pública Méx. 1990; 32(2):113-117.

15. Donabedian A. Priorities for progress in quality assesment and monitoring. Salud Publica Mex. 1993; 35(1):94-97.

16. Uchimura KY, Bosi MLM. Qualidade e subjetividade na avaliação de programas e serviços de saúde. Cad Saude Publica 2002; 18(6):1561-1569. 
17. Villela WV, Araújo EC, Ribeiro AS, Cuginotti AP, Hayana ET, Brito FC, Ramos LR. Desafios da Atenção Básica em Saúde: a experiência de Vila Mariana, São Paulo, Brasil. Cad Saude Publica 2009; 25(6):1316-1324.

18. Cotta RMM, Marque ES, Maia TM, Azeredo CM, Schott M, Franceschini SCC, Priori SE. A satisfação dos usuários do Programa de Saúde da Família: avaliando o cuidado em saúde. Sci. med. 2005; 15(4):227-234.

19. Ronzani TM, Silva CM. O Programa de Saúde da Família segundo profissionais de saúde, gestores e usuários. Cien Saude Colet 2008; 13(1):23-34.

20. Gea MT, Hernán-García M, Jimenéz-Martín JM, Cabrera A. Opinión de los usuarios sobre la calidad del Servicio de Urgencias del Centro MédicoQuirúrgico Del Hospital Virgen de las Nieves. Rev. calid. asist 2001; 16:37-44.

21. Medeiros FA, Araújo-Souza GC, Barbosa AAA, Clara-Costa IC. Acolhimento em uma Unidade Básica de Saúde: a satisfação do usuário em foco. Rev. salud pública 2010; 12(3):402-413.

22. Azevedo ALM, Costa AM. A estreita porta de entrada do Sistema Único de Saúde (SUS): uma avaliação do acesso na Estratégia de Saúde da Família. Interface Comun Saúde Educ 2010; 14(35):797-810.

23. Trad LAB, Bastos ACS, Santana EM, Nunes MO. Estudo etnográfico da satisfação do usuário do Programa de Saúde da Família (PSF) na Bahia. Cien Saude Colet 2002; 7(3):581-589.

24. Almeida C, Macinko J. Validação de uma metodologia de avaliação rápida das características organizacionais e do desempenho dos serviços de atenção básica do sistema único de saúde (SUS) em nível local. Brasília: Organização Pan- Americana da Saúde; 2006.

25. Schimith MD, Lima MADS. Acolhimento e vínculo em uma equipe do Programa Saúde da Família. Cad Saude Publica 2004; 20(6):1487-1494.

26. Brasil. Ministério da Saúde (MS). Secretaria de Políticas de Saúde. Departamento de Atenção Básica. Parâmetros para programação das ações básicas de saúde. Brasília: MS; 2001.

27. Aldana JM, Piechulek H, Al-Sabir A. Client satisfaction and quality of health care in rural Bangladesh. Bull. World Health Organ 2001; 79(6):512-517.

28. González AR, Jodra VM. Calidad percibida por dos poblaciones adscritas a dos Centros de Salud de la provincia de Cuenca. Rev Esp Salud Pública 2000; 74(3):275-286.
29. Ogden J, Bavalia K, Bull M, Frankum S, Goldie C, Gosslau M, Jones A, Kumar S, Vasant K. "I want more time with my doctor": a quantitative study of time and the consultation. J. Am. Board Fam. Pract. 2004; 21(5):479-483.

30. Singh H, Haqq ED, Mustapha N. Patients perception and satisfaction with health care professionals at primary care facilities in Trinidad and Tobago. Bull. World Health Organ. 1999; 77(4):356-360.

31. Ferri SMN, Pereira MJB, Mishima SM, Caccia-Bava MCG, Almeida MCP. As tecnologias leves como geradoras de satisfação em usuários de uma unidade de saúde da família. Interface Comun Saúde Educ 2007; 11(23):515-529.

32. Paixão NRD, Castro ARM. Grupo sala de espera: trabalho multiprofissional em Unidade Básica de Saúde. Bol. Saúde 2006; 20(2):71-78.

33. Souza ECF, Vilar RLA, Rocha NSPD, Uchoa AC, Rocha PM. Acesso e acolhimento na atenção básica: uma análise da percepção dos usuários e profissionais de saúde. Cad Saude Publica 2008; 24(Supl. 1):100-110

34. Brasil. Política Nacional de Medicamentos. Portaria No 3.916/MS/GM, de 30 de outubro de 1998. Diário Oficial da União 1998; 10 nov.

35. Souza RKT, Scholz MR. Utilização e avaliação dos serviços oferecidos por uma unidade básica de saúde do município de Maringá-PR. Saúde debate 1996; (52):35-41.

36. Brasil. Conselho Nacional de Secretários de Saúde. A saúde na opinião dos Brasileiros. 20a Edição. Brasília: CONASS; 2003.

37. Swarcwald CL, Leal MC, Gouveia GC, Souza WV.Desigualdades socioeconômicas em saúde no Brasil: resultados da Pesquisa Mundial de Saúde. Rev. bras. saúde matern. infant. 2005; 5(Supl. 1):1-22.

Artigo apresentado em 30/04/2012

Aprovado em 17/07/1012

Versão final apresentada em 30/08/2012 